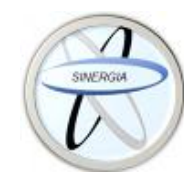

\title{
Endocarditis infecciosa
}

\author{
Infective endocarditis
}

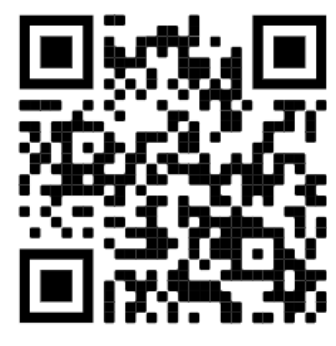

\author{
${ }^{1}$ Dr. Alberto Núñez Guerrero \\ Investigador independiente, San José, Costa Rica \\ (D) https://orcid.org/0000-0002-3611-3600
}

${ }^{2}$ Dra. Marilyn Campos Quesada Investigadora independiente, San José, Costa Rica

(1) https://orcid.org/0000-0001-6222-3769

${ }^{3}$ Dr. Daniel Molina Castaño

Investigador independiente, San José, Costa Rica

(C) https://orcid.org/0000-0003-0409-5839

RECIBIDO

CORREGIDO

ACEPTADO

$27 / 07 / 2020$

$25 / 08 / 2020$

$24 / 09 / 2020$

\section{RESUMEN}

La endocarditis infecciosa, es una patología poco usual que afecta principalmente válvulas nativas relacionadas a cuidados de la salud, se presenta con mayor frecuencia en adultos mayores de 60 años y en el sexo masculino. Sus factores predisponentes a lo largo del tiempo han ido modificándose, principalmente en países desarrollados, desplazando a la fiebre reumática. Son múltiples los microorganismos involucrados en la patología y su distribución a nivel global se ha mantenido estable, siendo las bacterias Cocos Gram-positivos las más frecuentes colonizadoras. Para que exista una infección debe haber bacteriemia en condiciones locales que permitan la proliferación e invasión de

$\mathbf{1}_{\text {Médico general, graduado }}$ de la Universidad de Ciencias Médicas (UCIMED), cod.MED16141. anunezg94@gmail.com

2 Médico general, graduada de la Universidad de Ciencias Médicas (UCIMED), cod. MED16060. marilyncampos94@hotmail.com

${ }^{3}$ Médico general, graduado

de la Universidad de (UCIMED), cod. MED16131 damorfl@hotmail.com endocardio. Se debe sospechar en todo paciente con fiebre asociada a síntomas constitucionales y factores de riesgo para endocarditis, los métodos diagnósticos de elección son los hemocultivos y ecocardiografía respectivamente. El tratamiento se fundamenta en la erradicación del microorganismo, antibiótico terapia de amplio espectro y se reserva la cirugía para casos que desarrollen complicaciones que solo puedan ser tratadas en sala de operaciones. La profilaxis antibiótica solo está recomendada en pacientes de riesgo.

PALABRAS CLAVE: endocarditis; fiebre; hemocultivo; antibióticos.

\section{ABSTRACT}

Infective endocarditis, is a rare disease that mainly affects native valves related to health care, occurs more frequently in adults over 60 years of age and in the male sex. Its predisposing factors over time have been changing, mainly in 
developed countries, displacing rheumatic fever. There are many microorganisms involved in the pathology and its distribution at a global level has remained stable, with the Gram-positive Cocos bacteria being the most frequent colonizers. For an infection to exist, there must be bacteremia under local conditions that allow endocardial proliferation and invasion. It should be suspected in all patients with fever associated with constitutional symptoms and risk factors for endocarditis, the diagnostic methods of choice are blood cultures and echocardiography respectively. The treatment is based on the eradication of the microorganism, antibiotic broad-spectrum therapy and surgery is reserved for cases that develop complications that can only be treated in the operating room. Antibiotic prophylaxis is only recommended in patients at risk.

KEYWORDS: endocarditis; fever; blood culture; anti-bacterial agents.

\section{INTRODUCCIÓN}

La endocarditis infecciosa es una afección séptica que predominantemente es de origen bacteriano, aunque existe la etiología fúngica, del endotelio valvular nativo del corazón, sin embargo, a su vez puede involucrar válvulas protésicas, así como dispositivo intracardiacos y el tejido endocárdico per se (1). Es una enfermedad seria que está asociada a una alta mortalidad y complicaciones a pesar de avances en su abordaje, su incidencia no ha bajado en los últimos 30 años $(1,2)$ y es el síndrome infeccioso amenazante para la vida más común después de la sepsis, neumonía y los abscesos intraabdominales (3).

Los cambios que han ocurrido en demografía a través de los años, así como patrones de resistencia antibiótica y cepas de patógenos más virulentos y los diferentes factores de riesgo en la actualidad provocan que el manejo de la endocarditis infecciosa sea un reto para el equipo que lo aborda $(1,4)$.

El objetivo principal de esta revisión literaria es dar a conocer integralmente diferentes puntos de interés para el profesional en salud que se enfrente con el síndrome infeccioso en cuestión, con la finalidad que sea de sospecha siempre que fuere menester en pacientes como descritos en este artículo y con un impacto significativo en la disminución de la mortalidad que estos acarrean; como susodicho, se detallan las bases de la patogenia, epidemiología y etiología de la enfermedad, a su vez, se hace especial énfasis en las manifestaciones clínicas y los diversos métodos diagnósticos y por último, pero de suma importancia, los avances más recientes en tratamiento, tanto antibiótico (clasificándolo según el patógeno causante) hasta quirúrgico, abarcando las indicaciones más relevantes, aunado a esto se brindan recomendaciones para profilaxis en pacientes de riesgo.

\section{MÉTODO}

Para la realización de este artículo de revisión, la búsqueda y localización de información se realizó a través de la base de datos DynaMed y Uptodate, capítulos de los libros Harrison y Braunwald de Medicina Interna y Cardiología, respectivamente, así como de artículos revisados en PubMed (Biblioteca Nacional de Medicina de los Estados Unidos), además basados en las guías de la Sociedad Europea de 
Cardiología, Asociación Americana del Corazón y del Colegio Americano de Cardiología. Los artículos y libros de texto que se utilizaron no tienen una antigüedad mayor a 5 años, utilizando los siguientes términos para búsqueda, tanto en idioma inglés como en idioma español: endocarditis infecciosa, diagnóstico endocarditis infecciosa, tratamiento endocarditis infecciosa, abordaje endocarditis infecciosa.

\section{EPIDEMIOLOGÍA}

La endocarditis infecciosa es una patología poco frecuente, se reportan aproximadamente de 3-10 casos por 100.000 habitantes, lo cual tiende a aumentar hasta 14.5 por 100.000 habitantes en pacientes que superan el sexto decenio de vida $(5,6)$. Originalmente la enfermedad predominaba pacientes jóvenes, esto en relación a la alta incidencia de valvulopatía reumática y enfermedades cardiacas congénitas, sin embargo, estos factores predisponentes han ido cambiando a lo largo del tiempo, principalmente en países desarrollados, debido al mejor reconocimiento y manejo de la faringitis estreptocócica, aumento de la esperanza de vida y mayor número de procedimientos invasivos con el advenimiento de la medicina moderna. Actualmente el consumo de drogas intravenosas, las valvulopatías degenerativas, principalmente la de la válvula mitral y el uso de dispositivos intracardiacos han desplazado en prevalencia a los que fueron los factores predisponentes clásicos (5-9).

Es más frecuente en el sexo masculino $(5,6)$, se estima que $25-35 \%$ de los casos se presentan en válvulas nativas relacionados a los cuidados de la salud y $16-30 \%$ en prótesis valvulares (8). A pesar de los avances en el manejo médico, aún representa una tasa de mortalidad del $20 \%$ (7).

\section{ETIOLOGÍA}

Una amplia gama de microorganismos como bacterias y hongos, pueden ocasionar la endocarditis infecciosa $(5,10)$. La distribución a nivel global se ha mantenido similar, con predominio de cocos gram positivos (estreptococos, estafilococos, y especies de enterococos) $(1,10)$. Los estreptococos y estafilococos son los principales agentes comunitarios (5).

- Especies de estreptocos: el grupo viridans son los microorganismos predominantes en endocarditis infecciosa $(\mathrm{EI}), \quad(5,10)$ ocasionando alrededor del $45-60 \%$ de los casos (5). La presentación típica en este grupo es la subaguda (síntomas presentes por semanas hasta meses) con síntomas frecuentes como fatiga, fiebre leve, y sudoración nocturna (10). Usualmente estos microorganismos se encuentran en la cavidad oral de los seres humanos y causan infecciones indolentes $(1,5,10)$. Dentro del grupo viridans se encuentran sanguis, oralis (mitis), salivarius, mutans, intermedius, anginosus y constellatus $(10,11)$. El grupo viridans constituye la principal causa de infecciones de las válvulas nativas adquiridas en la comunidad, siendo un sustrato común para estos microorganismos la valvulopatía reumática $(1,10)$.

Los estreptococos $\beta$ hemolíticos tienen una prevalencia menor del $10 \%$ de El, y generalmente causan una presentación aguda a diferencia del grupo viridans, siendo los consumidores de drogas intravenosa y adultos mayores dos grupos de riesgo $(5,10)$. Las 
complicaciones en este grupo es frecuente, y estas implican la destrucción de la válvula e infección en sitios distantes, usualmente musculoesqueléticos (10).

Streptococcus gallolyticus se encuentra en tubo digestivo y si se aísla en hemocultivo (ya sea relacionado a una endocarditis infecciosa o no), debe realizarse una exploración por si existe lesión gastrointestinal subyacente, como cáncer de colon $(1,10,11)$.

El Streptococcus pneumoniae hoy en día es una causa poco frecuente de El, con una prevalencia del $1-3 \%$ de los casos. $(5,10)$ La presentación clínica de este microorganismo es un síndrome agudo con destrucción de válvulas $(10,11)$, con una predilección de la válvula aórtica $(5,11)$. Puede asociarse a meningitis como otras complicaciones intracraneales $(2,10,11)$. Estos pacientes usualmente requieren cirugía para las complicaciones relacionadas a las válvulas (10).

- Especies de estafilococos: son el segundo grupo de cocos grampositivos como causas de El. El S.aureus es una causa frecuente de endocarditis tanto de válvulas nativas como protésicas $(1,10,12)$. La presentación es aguda, con toxicidad sistémica considerable (1,2,5,10-12). La infección de corazón derecho, especialmente en válvula tricúspide (consumidores de drogas por vía intravenosa), tiene una tasa de resolución mucho más alta que la izquierda, y una mortalidad baja (a menos que existe infección bilateral) (10). La tasa de El por S.aureus está aumentando, en parte, a la exposición de procedimientos sanitarios invasivos $(10,11)$.
Los estafilococos coagulasa negativos tienen una presentación subaguda y son patógenos frecuentes de infección de válvulas protésicas $(10,11)$. En una minoría de los casos de El, pueden afectar las válvulas nativas, esto en relación al uso de catéteres y procedimientos invasivos (10-12). Staphylococcus epidermidis es la especie que se identifica con más frecuencia en El y bacteremia $(10,11)$. Estos organismos son frecuentes en la contaminación de hemocultivos, por lo que debe tomarse varias muestras para diferenciar entre una contaminación y una septicemia (10).

- Especies de enterococo: la edad se correlaciona con el desarrollo de endocarditis en este grupo $(1,5,10)$, además de enfermedades crónicas y enfermedades valvulares de fondo. (1) La mayoría de casos es por causa de Enterococcus faecalis, y se asocia a anomalías del tracto genitourinario $(5,10,11)$. Recientemente, las especies de enterococos asociados a la atención sanitaria y catéteres venosos centrales han contribuido a la predisposición de la infección $(10,11)$.

- Microorganismos HACEK: son bacilos gram negativos que comprenden especies de Haemophilus (excepto $\mathrm{H}$. influenzae), Aggregatibacter actinomycetemcomitans y Aggregatibacter aphrophilus; Cardiobacterium hominis, Eikenella corrodens y Kingella kingae y $\mathrm{K}$. denitrificans $(1,5,10)$. Estos microorganismos colonizan el tracto respiratorio superior, $(1,5,10)$ con una presentación de El subaguda adquirida en la comunidad (10). La gran mayoría 
de estos microorganismos necesitan varios días de incubación en los hemocultivos (10). Es frecuente la embolia cerebral $u$ otras áreas sistémicas debido a la formación de grandes vegetaciones por un retraso diagnóstico, usualmente por el curso clínico indolente $(5,10)$.

- Bacilos aerobios gramnegativos: es infrecuente la El por estos microorganismos. Este grupo comprende Escherichia Coli, Klebsiella spp., Enterobacter spp., Pseudomonas spp. y otros. Usualmente la presentación de El es aguda y algunas veces se ha asociado a toxicidad sistémica (incluida sepsis y sus complicaciones) (10).

- Hongos: causan alrededor del $10 \%$ de casos de El, siendo unos microorganismos muy infrecuentes $(5,10)$. Su diagnóstico puede dificultarse debido a que algunos de estos microorganismos no crecen en los medios de cultivos habituales (10). La mayoría de estas infecciones son causadas por Candida spp, $(1,5,10)$, son una causa significativa en pacientes inmunocomprometidos(1) y con válvulas protésicas $(1,10)$. Estas suelen aparecer debido a una infección de un catéter venoso central (10). Debido a que las complicaciones son frecuentes, las intervenciones quirúrgicas son habituales, especialmente en infecciones por mohos como Aspergillus spp $(2,10)$.

- Endocarditis con cultivo negativo: los organismos que pertenecen a este grupo son los hongos, Coxiella burnetii, Bartonella spp., Brucella spp., Tropheryma whippelii y Legionella spp. (1,10-12) La mayoría de los casos que se clasifican como endocarditis infecciosa con hemocultivo negativo, el patógeno no se aísla por una reciente exposición a un antimicrobiano con actividad supresora o la eliminación del patógeno (2,10-12). Además, muchos patógenos no se aíslan en el hemocultivo habitual o crecen lentamente por lo que no se detecta a tiempo $(2,10,11)$.

\section{PATOGENIA}

La infección endocárdica va a depender de la presencia de bacteriemia en condiciones que permitan la proliferación e invasión local. El endotelio intacto usualmente es resistente a la mayoría de procesos infecciosos y a la formación de trombos, sin embargo, este se puede lesionar por diferentes mecanismos, ejemplo de esto son los lados de baja presión de una lesión cardiaca o sitios de gran impacto por chorros turbulentos a gran velocidad, existen lesiones anatómicas predisponentes, las más comunes alteraciones de válvulas cardiacas $(1,2,9,13)$.

Estas lesiones pueden producir acumulaciones de plaquetas y fibrina (endocarditis trombótica no bacteriana) que permiten a microorganismos virulentos causar lesiones directas, permitiendo que durante las bacteriemias transitorias que caracterizan a la enfermedad, exista la adhesión de patógenos infecciosos $(1,5,8)$. Diversos tipos de microorganismos entran al torrente sanguíneo desde las mucosas, piel o sitios de infección local, no obstante, hay excepciones de microorganismos con alta virulencia, como es el caso de $S$. aureus y $S$. bovis que tienen la capacidad de adherirse de manera directa, esta característica está relacionada a las moléculas de adhesina superficiales, denominados en conjunto componentes microbianos superficiales 
reconocedores de moléculas de adhesión de la matriz, aunado a esto participan proteínas que funcionan como facilitadoras de unión $(8,13)$. Cuando los microorganismos logran evadir la actividad bactericida del suero, se forman colonias densas, depósitos plaquetarios y estados procoagulantes localizados, que al combinarse con depósitos de fibrina y células de la inflamación forman la vegetación, dentro de esta las bacterias carecen de actividad metabólica y tienen cierta resistencia antibiótica $(5-8,13)$.

\section{FACTORES DE RIESGO}

La endocarditis infecciosa afecta con más frecuencia el sexo masculino, sin embargo, esta cifra está desapareciendo debido a un aumento de contactos con los servicios sanitarios en la población femenina (10). Cualquier patología estructural del corazón, sea adquirida 0 congénita, puede predisponer a una endocarditis infecciosa $(4,10)$. La enfermedad reumática cardiaca en países en desarrollo, es la causa más común que predispone a una $\mathrm{El}$, mientras que en los países desarrollados la prevalencia ha disminuido en las últimas décadas $(4,8,10)$. Las válvulas cardiacas protésicas y dispositivos cardiacos son factores de riesgo significativos de El, además de que su utilización ha aumentado en la última década.

Otros factores de riesgo importantes son el uso de drogas intravenosas y el estado inmunológico del paciente, como los portadores del virus de inmunodeficiencia humana $(4,5,8,10,12)$.

\section{MANIFESTACIONES CLÍNICAS}

La insuficiencia mitral asociada a prolapso mitral degenerativo es el trastorno predisponente más frecuente de El. (10) En relación a los síntomas, la fiebre (mayor a 38 grados $\mathrm{C}$ ) es la manifestación más frecuente (hasta 95\% de los pacientes) (1,5,10-12). Sin embargo, puede estar ausente hasta un $20 \%$, siendo la edad avanzada, inmunodepresión y pacientes tratados con antibiótico terapia los factores causantes $(1,10)$.

Un $20-40 \%$ de los pacientes presentan síntomas menos frecuentes, como escalofríos, sudoración, tos, cefalea, nauseas, mialgias, artralgias y malestar general (10). En aquellos pacientes con una presentación subaguda o crónica, también se puede observar anorexia, pérdida de peso, artralgias y dolor abdominal, síntomas que pueden orientar al médico a un diagnóstico incorrecto, como tumores malignos $(1,10,11)$.

La insuficiencia cardiaca complica alrededor de un $50 \%$ de estos pacientes, siendo la complicación más frecuente de El. Es imprescindible su reconocimiento precoz, puesto que es la que tiene mayor impacto sobre el pronóstico y es el factor predictivo más importante de un mal resultado de tratamiento quirúrgico. Las alteraciones en los ruidos cardiacos, se encuentran hasta en un $80 \%$ de los pacientes $(1,5,10,12)$. Se puede auscultar un soplo definido, en particular del lado izquierdo (10).

Se puede identificar una anomalía neurológica central y pueden detectarse déficits focales compatibles con accidente cerebrovascular en 10 a $20 \%$ de los pacientes $(1,6,10,11)$. Otras complicaciones potenciales de $\mathrm{El}$ son convulsiones, hemorragia subaracnoidea, encefalopatía tóxica, entre otras $(6,10,11)$.

Actualmente, dado a los avances en el diagnóstico y tratamiento precoz, no se observan las manifestaciones periféricas clásicas de El. $(1,10,12)$ Las petequias son 
las más frecuentes, produciéndose en la conjuntiva, mucosa oral o extremidades $(6,10,11)$.

Las lesiones de Janeway son máculas hemorrágicas indoloras, que tienen una predilección por las plantas de los pies 0 manos $(1,5,6,10,11)$, debido a una secuela de embolia séptica periférica (10). Las hemorragias ungueales en astilla son lesiones lineales indoloras de color rojo oscuro, que se localizan en el lecho ungueal proximal $(6,10,11)$.

Los nódulos de Osler son lesiones nodulares, eritematosas, dolorosas, que suelen localizarse en la yema de los dedos y pies $(1,5,6,10,11)$, causados por depósito de complejos inmunitarios y vasculitis localizada $(6,10)$. Las manchas de Roth son hemorragias retinianas con un centro de fibrina coagulada $(1,6,10)$, que también están relacionadas con la vasculitis mediada por complejos inmunitarios (10). Los nódulos de Osler y manchas de Roth pueden aparecer en otros trastornos, como Lupus eritematoso sistémico, leucemia y endocarditis no bacteriana. Aparte de petequias y hemorragias conjuntivales, estos hallazgos se detectaron en menos del $10 \%$ de los pacientes (10).

\section{DIAGNÓSTICO}

El clínico siempre debe sospechar una endocarditis de origen infecciosa en todo paciente con fiebre asociada a síntomas constitucionales y factores de riesgo para endocarditis (1). Por otro lado, se debe tener un bajo umbral de sospecha en paciente con presentaciones atípicas, como los adultos mayores e inmunocomprometidos, quienes, contrario a la población joven sana, es menos común la presencia de fiebre, que, por lo contrario, está presente hasta en un $90 \%$ de casos típicos de endocarditis $(2,8)$.
Los métodos de diagnósticos de primera elección, tanto de laboratorio como de imagen son los hemocultivos y la ecocardiografía, respectivamente.

Los hemocultivos son el método diagnóstico por laboratorio más importante ya que la mayoría de pacientes con endocarditis presentarán positivización de dicho estudio microbiológico, sin embargo, debido a la existencia de endocarditis con hemocultivos negativos, existen otros estudios de laboratorio más específicos para agentes que no positivizan en hemocultivos convencionales, entre ellos están los anticuerpos séricos para detección de Coxiella burnettii, Bartonella spp, Brucella spp...; histopatología en casos de obtener una biopsia o la pieza valvular infectada, en cuyo escenario ayuda a detectar microorganismos como Streptococcus, Staphylococcus, hongos, Tropheryma whipplei...; de igual manera otros como la reacción en cadena de polimerasa, inmunohistología, autoinmunihistoquímica y demás $(2,4,8,10)$.

Aunque no son diagnósticos, otros estudios de laboratorios que pueden estar alterados en la endocarditis infecciosa son: hemograma (anemia y leucocitosis), elevación de marcadores inflamatorios (proteína C reactiva y velocidad de eritrosedimentación), factor reumatoide positivo y microhematuria. Por otro lado, en un electrocardiograma se pueden manifestar trastornos de la conducción cardíaca, que traduce extensión de la infección al sistema de His-Purkinje, e inclusive isquemia cardíaca por fenómenos embólicos a las arterias coronarias $(1,2,8)$.

El ecocardiograma es la técnica radiodiagnóstica de elección y juega un rol fundamental en el diagnóstico, tratamiento y monitoreo de paciente con dicha patología; es útil para demostrar presencia de 
vegetaciones en las válvulas cardíacas, abscesos, dehiscencia valvular, soplos, y se deje garantizar su realización en todo paciente con sospecha de endocarditis $(3,8,10,14)$. El primer paso es realizar un ecocardiograma transtorácico con posterior progresión a un ecocardiograma transesofágico, el cual es más sensible que el primero para detectar lesiones más pequeñas y complicaciones locales como abscesos intracardíacos; en paciente con válvulas protésicas 0 dispositivos intracardíacos el estudio de elección es el ecocardiograma transesofágico sobre el transtorácico $(10,13,14)$. La AHA recomienda la realización de ecocardiograma transtorácico en todos los casos sospechados de endocarditis infecciosa (3).

A manera de englobar la clínica y estudios complementarios, existen los criterios de Duke, los cuales dictan los estatutos a seguir para diagnosticar una endocarditis, se dividen en criterios mayores y menores:

\section{Criterios mayores:}

1- Hemocultivos positivos por organismos típicos para endocarditis o hemocultivos persistentemente positivos a pesar de terapia antimicrobiana.

2- Vegetación (es) valvular (es) o dehiscencia de válvula protésica en ecocardiograma.

3- Hemocultivo positivo por C. burnettii o título de anticuerpo lgG antifase 1 igual o mayor a 1:800.

Los criterios menores son: factores predisponentes, fiebre, secuelas vasculares (eventos embólicos), consecuencias inmunológicas (nódulos de Osles y manchas de Roth), o hemocultivos positivos por microorganismos atípicos para endocarditis.
El diagnóstico definitivo se realiza con cualquiera de las siguientes combinaciones:

- Dos criterios mayores

- Un criterio mayor y tres criterios menores

- Cinco criterios menores.

Un diagnóstico probable se realiza en el contexto de: un criterio mayor y uno menor; o tres criterios menores $(2,7,8,10,14)$.

\section{TRATAMIENTO}

El tratamiento de la endocarditis infecciosa se fundamenta en la erradicación del microorganismo responsable, siguiendo pautas según la sensibilidad antibiótica demostrada en un antibiograma. En casos de alta sospecha de endocarditis y en un paciente en condición clínica deteriorada (shock séptico), se deberá iniciar antibioticoterapia empírica sin esperar el resultado de un antibiograma, siempre dirigido al patógeno más probable de causar la infección y haciendo diferencia de duración de tratamiento si la infección es en válvulas nativas o protésicas $(6,10)$. Es importante recalcar que los antibióticos a usar deberán ser bactericidas y no bacteriostáticos debido a que el sistema de defensa del huésped es de poca ayuda $(2,8)$.

- Streptococcus oral y $S$. bovis sensibles a penicilina: este grupo se cataloga como sensibles debido a que tienen una concentración mínima inhibitoria (CMI) igual o menor a 0.125 $\mathrm{mg} / \mathrm{L}$. En casos no complicados, se puede administrar penicilina 0 ceftriaxona junto con gentamicina durante un curso de 2 semanas. En pacientes alérgicos a beta-lactámicos en quienes no se puede realizar desensibilización, se usará vancomicina como antibiótico de elección. Las dosis y 
duración a usar, según cada antibiótico y régimen, son las siguientes $(2,3,13)$ :

1. Régimen estándar de 4 semanas en pacientes >65 años o con alteración de la función renal, o duración de 6 semanas en pacientes con infección de válvulas protésicas: penicilina G 12-18 millones de unidades endovenosas en 46 dosis o continua, o, ceftriaxona 2 g/día endovenosos o intramusculares en una dosis.

2. Régimen estándar de 2 semanas en pacientes con infección de válvulas nativas y función renal conservada: penicilina $\mathrm{G}$ o ceftriaxona con las dosis mencionadas previamente, más, gentamicina $3 \mathrm{mg} / \mathrm{kg} /$ día endovenoso o intramuscular en una dosis. La adición de gentamicina a la penicilina ejerce una acción sinergística, el mismo efecto occure con penicilina y ceftriaxona.

- Streptococcus oral y $S$. bovis resistentes a penicilina: estos patógenos pueden tener resistencia intermedia si la CMI está entre $0.25-2$ $\mathrm{mg} / \mathrm{L}$ o completamente resistentes si la $\mathrm{CMl}$ es igual o mayor a 4 . En el tratamiento estándar se pueden utilizar los mismos medicamentos y dosis que el tratamiento estándar de 2 semanas descrito en el apartado previo, con la excepción de la penicilina $G$ en la que la dosis es aumentada a 24 millones de unidades en este escenario. En pacientes alérgicos a beta-lactámicos se usa vancomicina a unas dosis de 30 $\mathrm{mg} / \mathrm{kg} /$ día. La duración del tratamiento, sea alérgico o no el paciente, es de 4 semanas, excepto si involucra válvulas protésicas lo cual aumenta la duración a 6 semanas por la poca penetración de medicamento en este tipo de tejido. La gentamicina se usa por las dos primeras semanas de tratamiento $y$ posteriormente se suspende $(2,4)$.

- Streptococcus pneumoniae, Streptococcus beta-hemolíticos del grupo A, B, C y G: en la era postantibiótico, la endocarditis infecciosa por S. pneumoniae es infrecuente y está asociada a meningitis en un $30 \%$ de los casos. Afín a los grupos descritos anteriormente, el tratamiento es similar en cepas tanto sensibles como resistentes. En casos de endocarditis con meningitis concomitante, el uso de penicilina debe ser evitado por su poca penetración en líquido cefalorraquídeo y será reemplazada por ceftriaxona o cefotaxima más vancomicina $(2,4,10,13)$.

- Staphylococcus spp: los antibióticos involucrados en los regímenes contra este género bacteriano son aquellos como la vancomicina, oxacilina, rifampin y gentamicina, entre otros. Las combinaciones entre ellos varían de acuerdo a la naturaleza de las válvulas afectadas, así como la duración de tratamiento, sin embargo, las fuerzas y dosis se mantienen. El régimen de elección para endocarditis en válvulas nativas y con un agente bacteriano sensible es: oxacilina 12g/día intravenoso en 4-6 dosis durante 4-6 semanas; régimen de elección en válvulas nativas en pacientes alérgicos y/o agente resistente: vancomicina 30-60 $\mathrm{mg} / \mathrm{kg} /$ día intravenosos en 2-3 dosis durante 4-6 semanas. En prótesis valvulares se utilizan combinaciones de antibióticos:

1. Régimen para agente sensible: oxacilina (misma dosis previa) con rifampin 900- 
$1200 \mathrm{mg}$ endovenoso $\mathrm{u}$ oral en 2-3 dosis, más, gentamicina $3 \mathrm{mg} / \mathrm{kg} / \mathrm{día}$ endovenoso 0 intramuscular en 1-2 dosis, por 6 o más semanas, excepto el aminoglucósido que se emplea durante 2 semanas.

2. Régimen para agente resistente 0 paciente alérgico a penicilina: vancomicina $\quad 30-60 \quad \mathrm{mg} / \mathrm{kg} / \mathrm{día}$ intravenosos en 2-3 dosis, el resto del tratamiento es igual al de agente sensible en válvula protésicas $(2,10)$.

Otros microorganismos que caben mencionar son el grupo HACEK y Enterooccus spp. Los primeros son bacilos gram negativos fastidiosis de crecimiento lento y que cuya MIC no es fácil de interpretar (3) y el tratamiento de primera elección es terapia antibiótica con ceftriaxona 2 g/día por 4 semanas en válvulas nativas y durante 6 semanas en prótesis valvular $(2,7)$.

El grupo de Enterococcus spp. ocupan el tercer lugar en cuanto a etiología de endocarditis, siendo $E$. faecalis y E. faecium los más frecuentemente aislados $(3,8,10)$. En cepas sensibles a penicilina, el tratamiento de elección abarca el uso de penicilina $\mathrm{G} o$ ampicilina (o amoxicilina) más gentamicina. Los aminoglucósidos no deben ser usados si existe una CMI mayor a 500 $\mathrm{mg} / \mathrm{L}$, en estos casos se debe usar una terapia alterna con estreptomicina $(2,13)$.

\section{TRATAMIENTO QUIRÚRGICO}

Hasta un $50 \%$ de pacientes con endocarditis desarrollan complicaciones que solo pueden ser resueltas en una sala de operaciones. Entre las indicaciones para cirugía se encuentran: falla cardíaca descompensada, infección local que no ha podido ser controlada (absceso miocárdico), escisión de grandes vegetaciones para prevenir embolismo y sepsis persistente a pesar de antibioticoterapia (1-3,10). A pesar de haber indicaciones definidas teóricamente, en la práctica se debe sopesar las comorbilidades del paciente, riesgo quirúrgico y el estado clínico del paciente. A su vez, en el transoperatorio es útil el ecocardiograma para localizar de manera exacta lesiones infecciosas y así guiar el acto quirúrgico además de evaluar resultados y ayudar en el seguimiento postoperatorio temprano $(3,10)$. Las indicaciones de cirugía cardiovascular se pueden englobar a grosso modo en complicaciones de la enfermedad 0 presentaciones severas de la misma, como las siguientes: pacientes descompensados de insuficiencia cardíaca, pacientes con bacteriemia persistente posterior a 5-7 días de antibiótico y se hayan excluido otras fuentes de infección, embolismos recurrentes, endocarditis causada por hongos 0 microorganismos altamente resistentes, entre otras $(1-3,7,8,10)$.

\section{PROFILAXIS ANTIBIÓTICA}

Luego de numerosos debates acerca de la profilaxis antibiótica para la endocarditis, actualmente las guías de manejo médico, recomiendan restringir su uso para pacientes que presenten un mayor riesgo, esto debido a que el tratamiento antimicrobiano se limita a prevenir un pequeño número de casos. Los pacientes de alto riesgo pueden agruparse en 3 categorías $(1,2,6,12,13,15)$ :

- Pacientes portadores de válvula protésica o material protésico usado para valvuloplastia. También aplica a las prótesis valvulares transcatéter y los aloinjertos.

- Pacientes con endocarditis infecciosa previa. 
- Pacientes con cardiopatías congénitas cianóticas no tratadas y aquellos con shunts posoperatorios paliativos, conductos u otras prótesis. Después de la reparación quirúrgica sin defectos residuales se recomienda profilaxis durante los primeros 6 meses después del procedimiento hasta que se produzca endotelización del material protésico.

Aunque algunas guías recomiendan profilaxis para los receptores de trasplante cardiaco que desarrollen valvulopatía cardíaca, no existen evidencias sólidas que lo respalden (2). La antibioticoterapia recomendada para procedimientos dentales en los que exista manipulación de la mucosa gingival, región periapical del diente y perforaciones de la mucosa oral, así como procedimientos quirúrgicos sobre zonas infectadas 0 drenaje de abscesos en pacientes de alto riesgo es: amoxicilina o ampicilina $2 \mathrm{~g}$ vía oral de 30-60 min antes del procedimiento, si existe alergia a penicilina, se recomienda el uso de clindamicina $600 \mathrm{mg}$ vía oral o intravenosa $30-60 \mathrm{~min}$ antes del procedimiento $(1,2,6,12,13)$. La profilaxis antibiótica sistemática no está recomendada en procedimientos no dentales. Solo es necesario cuando se realicen procedimientos invasivos en el contexto de una infección $(2,6,12)$.

\section{CONCLUSIÓN}

La endocarditis infecciosa es una patología excepcional debido a la amplia variedad cualidades que la caracterizan y su comportamiento a través del tiempo, a pesar de ser conocida como una enfermedad de baja prevalencia su padecimiento se asocia a altas tasas de mortalidad y morbilidad tanto intra como extra hospitalaria, por lo que se aconseja a los profesionales de la salud conocer sus patogenia y los principales microorganismos causales, para poder correlacionar con las manifestaciones clínicas en pacientes con alto índice de sospecha.

Se recomienda utilizar como herramienta diagnóstica los criterios de Duke, siendo la toma de hemocultivos y la ecocardiografía los pilares diagnósticos, esto con el fin de iniciar antibioticoterapia lo más pronto posible y estratificar pacientes en los que la cirugía temprana ofrezca mayor beneficio, a su vez se insta a los médicos utilizar profilaxis antibiótica en pacientes de alto riesgo en los que sean sometidos a tratamientos con manipulación gingival. Se concluye y espera que, dada a conocer información indispensable para la sospecha y manejo integral (inicial hasta definitivo y multidisciplinario) del síndrome abordado en este documento, se encuentre lugar en el abanico de diagnósticos diferenciales al abordar un paciente, con un bajo umbral de sospecha para que esta sea temprana, y cuando existan bases que lo sustenten, para así brindar el mejor manejo posible con el fin de tener un impacto positivo y significativo en la mortalidad de quienes se les brinda atención galena.

\section{REFERENCIAS}

1. Yang E, Frazee B,W. Infective Endocarditis. Emerg Med Clin N Am. [internet] 2018 [citado 22 febrero 2020] 36(4): 645-663. Disponible en: https://doi.org/10.1016/j.emc.2018.06.002 
2. Habib G, Lancellotti $P$, Antunes MJ, Bongiorni MG, Casalta JP, Del Zotti $F$ et al. ESC Guidelines for the management of infective endocarditis. EHJ. [internet] 2015 [citado 22 febrero 2020] 36:3075-3123. Disponible en: https://doi.org/10.1093/eurheart//ehv319

3. Baddour LM, Wilson WR, Bayer AS, Fowler VG, Tleyjeh IM, Rybak MJ et al. Infective Endocarditis in Adults: Diagnosis, Antimicrobial Therapy, and Management of Complications. Circulation.[internet] 2015 [citado 23 febrero 2020]135: 1435-1486. Disponible en: https://doi.org/10.1161/CIR.0000000000000296

4. Holland TL, Baddour LM, Bayer AS, Hoen B, Miro JM, Fowler VG. Infective endocarditis. Nature Reviews Disease Primers. [internet] 2016 [citado 23 febrero 2020] 1;2:16059. Disponible en: 10.1038/nrdp.2016.59

5. Conde mercado, JM.,Camacho Limas, CP.,Quintana Cuellar, M., De la Torre Saldaña, VA., Adán Brito, C. y Alonso Bello, CD.Endocarditis infecciosa. Rev Hosp Jua Mex, [internet] 2017 [citado 24 febrero 2020]. 84(3), 143-166. Doi: https://www.medigraphic.com/pdfs/juarez/ju-2017/ju173e.pdf

6. García-Arribas,D., Olmos, C., Vivas D y Vilacosta.Endocarditis infecciosa. Medicine. [internet] 2017 [citado 25 febrero 2020] 12 (40), 2380-2395. Doi:https://www.medicineonline.es/es-endocarditis-infecciosa-articulo$\underline{\mathrm{S} 0304541217302160}$

7. Klein M, Wang A. Infective endocarditis. J. Intensive Care Med. [internet] 2016 [citado 25 febrero 2020] 31(3): 151 163. Disponible en: https://doi.org/10.1177\%2F0885066614554906

8. Karchmer A.W. Endocarditis Infecciosa. En: Jameson JL, Kasper DL, Longo DL, et al, editores. Harrison principios de medicina interna. Vol1. 20va ed. México: McGraw-Hill; 2018. p. 921-933.

9. Muñoz P, Kestler M, De Alarcon A, Miro J.M, Bermejo J, Rodríguez-Abella $\mathrm{H}$ et al. Current Epidemiology and Outcome of Infective Endocarditis. Medicine (Baltimore) [internet] 2015 [citado 26 febrero 2020] 94(43): e1816. Disponible en: https://dx.doi.org/10.1097\%2FMD.0000000000001816

10. Baddour L.M, Freeman W.K, Suri R.M, Wilson W.R. Infecciones cardiovasculares. En: Mann D.L, Zipes D.P, Libby P, Bonow R.O, Tomaselli G.F, Braunwald E (Ed.). Braunwald tratado de cardiología. Vol 2. 11a ed. España: Elsevier, 2019 (pp.1483-1509).

11. Olmos Blanco, C. Epidemiología y pronóstico del shock séptico en la endocarditis infecciosa. Tesis doctoral. [internet] 2015 [citado 26 febrero] Universidad Complutense de Madrid.

12. Cahill T.J, Baddour L.M, Habib G, Hoen B, Salaun E, Petterson G.B et al. Callenges in Infective Endocarditis. JACC. [internet] 2016 [citado 1 marzo 2020] 69(3): 325-344. Disponible en: https://doi.org/10.1016/i.jacc.2016.10.066

13. Fernadez-Gerlinger MP, Mainardi JL. Endocarditis infecciosa: del diagnóstico al tratamiento. EMC-Tratado de Medicina.[internet] 2016 [citado 1 marzo 2020] 20(4): 1-7. Disponible en: https://doi.org/10.1016/S1636$\underline{5410(16) 80656-X}$

14. Vincent LL, Otto CM. Infective Endocarditis: Update on Epidemiology, Outcomes and Management. Current Cardiology Reports. [internet] 2018 [citado 1 marzo 2020] 16;20(10):86. Disponible en: https://www.ncbi.nlm.nih.gov/pubmed/30117004

15. Bolger AF. Preventing Endocarditis: No Rest for the Worrier.J Am Coll Cardiol. [internet] 2018 [citado 1 marzo 2020] 72(20): 2455-2456. Disponible en: https://doi.org/10.1016/j.jacc.2018.09.025 